\title{
PERIODIC ORBITS OF THE PLANAR ANISOTROPIC GENERALIZED KEPLER PROBLEM
}

\author{
JAUME LLIBRE ${ }^{1}$ AND CLÀUDIA VALLS ${ }^{2}$
}

\begin{abstract}
Many generalizations of the Kepler problem with homogeneous potential of degree $-1 / 2$ have been considered. Here we deal with the generalized anisotropic Kepler problem with homogeneous potential of degree -1 . We provide the explicit solutions of this problem on the zero energy level, and show that all of them are periodic.
\end{abstract}

\section{IntRoduCtion AND STATEMENT OF THE MAIN RESUlT}

The classical Kepler problem describes the motion of the two-body problem under the mutual gravitational attraction given by the Newtonian's universal law of gravitation.

In the papers $[2,9,10,11,13,14,16]$ different generalizations of the Kepler problem with homogeneous potential of degree $-1 / 2$ have studied, for instance generalizations to $n$-dimensional curved spaces, to charge quantization, to Euclidean Jordan algebra, to their integrability with Clifford algebras or with Lie algebras in quantum mechanics.

In the papers $[5,6,7,8]$ Gutzwiller generalized the Kepler problem to describe the motion of two-body in an anisotropic configuration plane with homogeneous potential of degree $-1 / 2$. Gutzwiller research wanted to find an approximation of the quantum mechanical energy levels for a chaotic system. Recently in the papers $[1,3,15]$ some dynamics and periodic orbits of this anisotropic Kepler problem were studied analytically.

Here we generalize the anisotropic Kepler problem from homogeneous potential of degree $-1 / 2$ to homogeneous potential of degree -1 . More precisely, the equations of motion of the planar anisotropic Kepler problem with homogeneous potential of degree -1 in Hamiltonian formulation are described by the Hamiltonian

$$
H=H\left(x, y, p_{x}, p_{y}\right)=\frac{1}{2}\left(p_{x}^{2}+p_{y}^{2}\right)-\frac{1}{(1+\varepsilon) x^{2}+y^{2}} .
$$

being $|\varepsilon|>0$ a small parameter which provides the anisotropy in the direction of the $x$-axis.

2010 Mathematics Subject Classification. Primary 34C05, 34A34, 34C14.

Key words and phrases. Anisotropic generalized Kepler problem, periodic orbits, McGehee coordinates. 
Note that the angular momentum for system (1) is not a first integral due to the fact that the anisotropy of the plane destroys the rotational invariance.

Our main result is the following one.

Theorem 1. We consider the generalized anisotropic Kepler problem with homogeneous potential of degree -1 given by Hamiltonian (1). Then:

(a) The energy level $H=0$ is diffeomorphic to the manifold $\mathbb{S}^{1} \times \mathbb{S}^{1} \times \mathbb{R}$.

(b) We provide the explicit expression of all orbits of the Hamiltonian system with Hamiltonian (1) on the energy level $H=0$, and all of them are periodic.

Theorem 1 is proved in section 2 .

\section{THE PROOF}

The Hamiltonian equations associated to the Hamiltonian (1) are

$$
\begin{aligned}
\dot{x} & =p_{x}, \\
\dot{y} & =p_{y}, \\
\dot{p}_{x} & =-\frac{2 x(1+\varepsilon)}{\left((1+\varepsilon) x^{2}+y^{2}\right)^{2}}, \\
\dot{p}_{y} & =-\frac{2 y}{\left((1+\varepsilon) x^{2}+y^{2}\right)^{2}} .
\end{aligned}
$$

Here the dot denotes derivative with respect to the time $t$. We note that the phase space of this Hamiltonian system is the set of points $\left(x, y, p_{x}, p_{y}\right)$ of $\left(\mathbb{R}^{2} \backslash\{(0,0)\}\right) \times \mathbb{R}^{2}$.

The key in the proof of Theorem 1 is to work in the so called McGehee coordinates, see $[12,4]$. Thus we consider the coordinate transformation $\left(x, y, p_{x}, p_{y}\right) \rightarrow(r, \theta, u, v)$ defined by

$$
\begin{aligned}
& r=\sqrt{x^{2}+y^{2}}, \\
& \theta=\arctan \left(\frac{y}{x}\right), \\
& u=r\left(-p_{x} \sin \theta+p_{y} \cos \theta\right), \\
& v=r\left(p_{x} \cos \theta+p_{y} \sin \theta\right) .
\end{aligned}
$$

and the rescaling of time

$$
d \tau=r^{-2} d t
$$

With this transformation, which is an analytic diffeomorphism in its domain of definition, system (2) becomes

$$
\begin{aligned}
& r^{\prime}=r v, \\
& \theta^{\prime}=u, \\
& u^{\prime}=-V^{\prime}(\theta), \\
& v^{\prime}=u^{2}+v^{2}+2 V(\theta),
\end{aligned}
$$


where

$$
V(\theta)=\frac{1}{(1+\varepsilon) \cos ^{2} \theta+\sin ^{2} \theta},
$$

and the prime denotes derivative with respect the new time $\tau$. The energy relation (1) in the new variables is

$$
H r^{2}=\frac{1}{2}\left(u^{2}+v^{2}\right)+V(\theta) .
$$

We note that the domain of definition of the differential system (3) are the points $(r, \theta, u, v)$ of $(0, \infty) \times \mathbb{S}^{1} \times \mathbb{R}^{2}$. Clearly we can extend this domain of definition to $r=0$, and thus we can study the solutions near the collision of the two bodies. So from now on the domain of definition of the differential system (3) are the points $(r, \theta, u, v)$ of $[0, \infty) \times \mathbb{S}^{1} \times \mathbb{R}^{2}$. We remark that McGehee [12] introduced these variables in order to study the collision manifold $r=0$.

From (4) the points of the zero energy level, $H=0$, satisfy

$$
u^{2}+v^{2}+2 V(\theta)=0 .
$$

For each $\theta \in \mathbb{S}^{1}$ we have a circle $\mathbb{S}^{1}$ for $(u, v)$, and since $r \in[0, \infty)$ we conclude that $H=0$ is diffeomorphic to $[0, \infty) \times \mathbb{S}^{1} \times \mathbb{S}^{1}$ in the coordinates $(r, \theta, u, v)$. Consequently the zero energy level in the variables $\left(x, y, p_{x}, p_{y}\right)$ is diffeomorphic to $(0, \infty) \times \mathbb{S}^{1} \times \mathbb{S}^{1}$. So statement (a) of Theorem 1 is proved.

The equations of motion (3) on the zero energy level $H=0$ reduce to

$$
\begin{aligned}
& r^{\prime}=r v, \\
& \theta^{\prime}=u, \\
& u^{\prime}=\varepsilon \frac{4 \sin (2 \theta)}{(2+\varepsilon(1+\cos (2 \theta)))^{2}}, \\
& v^{\prime}=0,
\end{aligned}
$$

Now these equations taking as independent variable the angular variable $\theta$ become

$$
\begin{aligned}
& \frac{d r}{d \theta}=\frac{r v}{u}, \\
& \frac{d u}{d \theta}=\varepsilon \frac{4 \sin (2 \theta)}{u(2+\varepsilon(1+\cos (2 \theta)))^{2}}, \\
& \frac{d v}{d \theta}=0 .
\end{aligned}
$$

We shall compute the solutions $(r(\theta), u(\theta), v(\theta))$ of system $(5)$.

It follows from $d v / d \theta=0$ that

$$
v(\theta)=v_{0} \quad \text { with } v_{0} \in \mathbb{R} .
$$

Moreover the solution of

$$
\frac{d u}{d \theta}=\frac{4 \varepsilon \sin (2 \theta)}{u(2+\varepsilon(1+\cos (2 \theta)))^{2}} \quad \text { with } u(0)=u_{0}
$$


is given by

$$
u(\theta)=\sqrt{2} \sqrt{\frac{2}{2+\varepsilon(1+\cos (2 \theta))}+\frac{u_{0}^{2}(1+\varepsilon)-2}{2(1+\varepsilon)}} .
$$

Clearly $u(\theta)$ is well-defined and $\pi$-periodic in the variable $\theta$.

Finally the solution of

$$
\frac{d r}{d \theta}=\frac{r(\theta) v(\theta)}{u(\theta)} \quad \text { with } r(0)=r_{0} .
$$

is given by

$r(\theta)=r_{0} \exp \left(\frac{v_{0} \sqrt{2} \sqrt{u_{0}^{2}(1+\varepsilon)^{2} \cos ^{2} \theta+\left(u_{0}^{2}+2 \varepsilon+u_{0}^{2} \varepsilon\right) \sin ^{2} \theta}}{\sqrt{u_{0}^{2}(1+\varepsilon)} \sqrt{2 u_{0}^{2}+\varepsilon\left(2+3 u_{0}^{2}+u_{0}^{2} \varepsilon\right)+\varepsilon\left(-2+u_{0}^{2}+u_{0}^{2} \varepsilon\right) \cos (2 \theta)}} P\left(u_{0}, \theta\right)\right)$,

where

$$
P\left(u_{0}, \theta\right)=-i\left(F\left(i \phi_{1}, k_{1}\right)+\varepsilon \Pi\left(1+\varepsilon, i \phi_{1}, k_{1}\right)\right),
$$

being

$$
F(\phi, m)=\int_{0}^{\phi}\left(1-m \sin ^{2} \theta\right)^{-1 / 2} d \theta=\int_{0}^{\sin \theta}\left[\left(1-t^{2}\right)\left(1-m t^{2}\right)\right]^{-1 / 2} d t
$$

the incomplete elliptic integral of the first kind and

$$
\begin{aligned}
\Phi(n, \phi, m) & =\int_{0}^{\phi}\left(1-n \sin ^{2} \theta\right)^{-1}\left(1-m \sin ^{2} \theta\right)^{-1 / 2} d \theta \\
& =\int_{0}^{\sin \phi}\left(1-n t^{2}\right)^{-1}\left[\left(1-t^{2}\right)\left(1-m t^{2}\right)\right]^{-1 / 2} d t,
\end{aligned}
$$

the incomplete elliptic integral of the third kind. Here

$$
\phi_{1}=\operatorname{arcsinh}\left(\sqrt{\frac{1}{1+\varepsilon}} \tan \theta\right), \quad k_{1}=\frac{2 \varepsilon+u_{0}^{2}(1+\varepsilon)}{u_{0}^{2}(1+\varepsilon)}, \quad n=1+\varepsilon .
$$

Note that using the equality $i \operatorname{arcsinh}(x)=\arcsin (i x)$ we get

$$
\begin{aligned}
-i F\left(i \phi_{1}, k_{1}\right) & \left.=-i \int_{0}^{i \frac{1}{\sqrt{1+\varepsilon}} \tan \theta}\left[\left(1-t^{2}\right)\left(1-k_{1} t^{2}\right)\right)\right]^{-1 / 2} d t \\
& =\frac{1}{\sqrt{1+\varepsilon}} \tan \theta \int_{0}^{1}\left[\left(1+s^{2} \frac{\tan ^{2} \theta}{1+\varepsilon}\right)\left(1+k_{1} s^{2} \frac{\tan ^{2} \theta}{1+\varepsilon}\right)\right]^{-1 / 2} d s,
\end{aligned}
$$

doing the change $t \rightarrow s$ given by

$$
t=\frac{i s}{\sqrt{1+\varepsilon}} \tan \theta
$$

Now define

$$
\begin{aligned}
P_{1}(\varepsilon, \theta, s) & =(1+\varepsilon) \cos ^{2} \theta+s^{2} \sin ^{2} \theta \\
P_{2}(\varepsilon, \theta, s) & =(1+\varepsilon) \cos ^{2} \theta+k_{1} s^{2} \sin ^{2} \theta \\
& =(1+\varepsilon) \cos ^{2} \theta+\frac{2 \varepsilon+u_{0}^{2}(1+\varepsilon)}{u_{0}^{2}(1+\varepsilon)} s^{2} \sin ^{2} \theta .
\end{aligned}
$$


Therefore we obtain

$$
-i F\left(i \phi_{1}, k_{1}\right)=\frac{\sqrt{1+\varepsilon}}{2} \sin (2 \theta) \int_{0}^{1}\left[P_{1}(\varepsilon, \theta, s) P_{2}(\varepsilon, \theta, s)\right]^{-1 / 2} d s .
$$

Note that this function is real, well-defined and $\pi$-periodic in the variable $\theta$. Proceeding analogously we get

$$
\begin{gathered}
\left.\left.-i \Pi\left(1+\varepsilon, i \phi_{1}, k_{1}\right)=-i \int_{0}^{i \frac{1}{\sqrt{1+\varepsilon}} \tan \theta}\left(1-(1+\varepsilon) t^{2}\right)\right)^{-1}\left[\left(1-t^{2}\right)\left(1-k_{1} t^{2}\right)\right)\right]^{-1 / 2} d t \\
=\frac{\tan \theta}{\sqrt{1+\varepsilon}} \int_{0}^{1}\left(1+s^{2} \tan ^{2} \theta\right)^{-1}\left[\left(1+s^{2} \frac{\tan ^{2} \theta}{1+\varepsilon}\right)\left(1+k_{1} s^{2} \frac{\tan ^{2} \theta}{1+\varepsilon}\right)\right]^{-1 / 2} d s,
\end{gathered}
$$

again with the change of variables (6). Using the notation of (7) and defining

$$
P_{3}(\varepsilon, \theta, s)=\cos ^{2} \theta+s^{2} \sin ^{2} \theta,
$$

we get

$-i \Pi\left(1+\varepsilon, i \phi_{1}, k_{1}\right)=\frac{\sqrt{1+\varepsilon} \sin (2 \theta) \cos ^{2} \theta}{2} \int_{0}^{1} P_{3}(\varepsilon, \theta, s)^{-1}\left[P_{1}(\varepsilon, \theta, s) P_{2}(\varepsilon, \theta, s)\right]^{-1 / 2} d s$.

Note that this function is real, well-defined and $\pi$-periodic in the variable $\theta$. Then we have that the solution $(r(\theta), u(\theta), v(\theta))$ is $\pi$-periodic. Hence all solutions in the zero energy level $H=0$ are $\pi$-periodic in the variable $\theta$ in the points $(r, \theta, u, v) \in[0, \infty) \times$ $\mathbb{S}^{1} \times \mathbb{R}^{2}$, and periodic in the time $t$ in the points $\left(x, y, p_{x}, p_{y}\right) \in\left(\mathbb{R}^{2} \backslash\{(0,0)\}\right) \times \mathbb{R}^{2}$. This completes the proof of statement (b) of Theorem 1.

\section{ACKNOWLEDGEMENTS}

The first author is partially supported by MINECO grants MTM2016-77278-P and MTM2013-40998-P, an AGAUR grant number 2014SGR-568, and the grant FP7PEOPLE-2012-IRSES 318999. The second author has been partially supported by FCT/Portugal through UID/MAT/04459/2013.

\section{REFERENCES}

[1] E.I. Abouelmagd, J.L.G. Guirao And J. Llibre, Periodic orbits of the planar anisotropic Kepler problem, to appear in Int. J. Bifurcation and Chaos.

[2] A. Ballesteros And F.J. Herranz, Maximal superintegrability of the generalized KeplerCoulomb system on N-dimensional curved spaces, J. Phys. A 42 (2009), no. 24, 245203, 12 pp.

[3] J. Casasayas And J. Llibre, Qualitative analysis of the anisotropic Kepler problem, Mem. Amer. Math. Soc. 52 (1984), no. 312, 115 pp.

[4] R. Devaney, Singularities in classical mechanical systems, Ergodic theory and dynamical systems I, (College Park, Md., 197980), pp. 211-333.

[5] M. Gutzwiller, Periodic orbits and alassical quantization conditions, J. Math. Phys. 12 (1971), 343-358.

[6] M. Gutzwiller, The anisotropic Kepler problem in two dimensions, J. Math. Phys. 14 (1973), $139-152$. 
[7] M. Gutzwiller, Bernoulli sequences and trajectories in the anisotropic Kepler problem, J. Math. Phys. 14 (1977), 806-823.

[8] M. Gutzwiller, Periodic orbits in the anisotropic Kepler problem, Classical mechanics and dynamical systems (Medford, Mass., 1979), pp. 69-90, Lecture Notes in Pure and Appl. Math. 70, Dekker, New York, 1981.

[9] P.E. KustaAnheimo, Motor integrals of a generalized Kepler motion, Celestial Mech. 6 (1972), $52-59$.

[10] P. Lounesto, Lie groups of motor integrals of generalized Kepler motion, Celestial Mech. 17 (1978), 207-213.

[11] A.E. Margolin And L.M. TomiL'CIK, The charge quantization condition in the generalized Kepler problem, (Russian) Vesci Akad. Navuk BSSR Ser. Fiz.-Mat. Navuk 1977, no. 3, 73-77.

[12] R. MCGEHEe, Singularities in classical celestial mechanics, Proceedings of the International Congress of mathematics, Helsinki.

[13] G. Meng, Generalized Kepler problems I. Without magnetic charges, J. Math. Phys. 54 (2013), 012109, 25 pp.

[14] G. Meng, Generalized Kepler problems and euclidean Jordan algebras, Geometry, integrability and quantization XVII, 7294, Bulgar. Acad. Sci., Sofia, 2016.

[15] C. Vidal, Periodic solutions for any planar symmetric perturbation of the Kepler problem, Celestial Mech. Dynam. Astronom. 80, 119-132.

[16] T. Vozmishcheva, The limit passage of space curvature in problems of celestial mechanics with the generalized Kepler and Hooke potentials, Astrophys. Space Sci. 361 (2016), no. 9, Paper No. 282, 7 pp.

${ }^{1}$ Departament de Matemàtiques, Universitat Autònoma de Barcelona, 08193 Bellaterra, Barcelona, Catalonia, Spain

E-mail address: jllibre@mat.uab.cat

2 Departamento de Matemática, Instituto Superior Técnico, Universidade de LisBoA, Av. Rovisco Pais 1049-001, Lisboa, Portugal

E-mail address: cvalls@math.ist.utl.pt 